\title{
An efficient technique for out-of-band power reduction for the eliminated CP-STC-shaped system for $5 \mathrm{G}$ requirements
}

\author{
Mohamed Yasin I. Afifi ${ }^{1}$, Mohamed S. Elbakry ${ }^{2}$,El-Sayed S. A. Said ${ }^{3}$, A. A. Ammar ${ }^{4}$ \\ ${ }_{1,3,4}$ Electrical Engineering Department, Faculty of Engineering, Al-Azhar University, Egypt \\ ${ }^{2}$ Electronics and Communications Engineering, Institute of Aviation Engineering and Technology, Egypt
}

\begin{abstract}
Article Info
Article history:

Received Oct 6, 2019

Revised Apr 8, 2020

Accepted Apr 18, 2020

\section{Keywords:}

$5 \mathrm{G}$

FBMC

OFDM

OOB

PAPR reduction

Pulse shaping

STC

ABSTRACT

The most dominant needs for the recent wireless mobile applications are higher bandwidth (BW) efficiency, higher energy efficiency higher quality of services (QOS). The main technique in $4 \mathrm{G}$ systems is OFDM but it suffers from some limitations such as large peak to average power ratio (PAPR), higher out-of-band (OOB) power radiation, and wasting bandwidth efficiency due to cyclic prefix (CP) extension. In his paper, these OFDM limitations will be reduced with low computational complexity compared to filter bank multicarriers (FBMC). The proposed scheme is based on symbol time compression (STC) for OFDM system. The proposed STC-Shaped system is achieved via interleaver-spreader and symbol shaper in the transmitter side in addition to equalization and combining processes in the receiver side. Comparative study between the proposed system and the conventional OFDM in case of additive white gaussian noise (AWGN) and COST 207 typical multipath fading channel will be presented. The numerical results show that the proposed STC-Shaped scheme reduces OOB significantly. The proposed scheme improves BER in multipath Rayleigh fading although it is without CP. Thus, the proposed system is more robust against inter symbol interference (ISI) compared to conventional OFDM system. Also, the numerical results show that the PAPR of the proposed system is decreased significantly and also, it is derived theoretically. Also, the proposed scheme overcomes CP extension, and hence increases the bandwidth (BW) efficiency. Finally, the computational complexity for the proposed scheme is derived and it has very low complexity compared to FBMC.
\end{abstract}

Copyright (C) 2020 Institute of Advanced Engineering and Science. All rights reserved.

Corresponding Author:

Mohamed Yasin I. Afifi,

Department of Electronics and Communications Engineering,

Al-Azhar University,

Al-Azhar University/Cairo - Nasr City Street, Permanent Camp, Egypt.

Email: mohamedyasin869@azhar.edu.eg

\section{INTRODUCTION}

As growth of wireless technologies, there is an increase in mobility, coverage, data rate and spectral efficiency and the main technique for 4G system is OFDM [1-2]. OFDM converts frequency selective fading channel into flat fading channel and hence OFDM has simple receiver [3, 4]. Nevertheless, OFDM has disadvantages, such as large spectrum side lobes, affecting high adjacent channel interference and OOB radiation $[5,6]$. In addition to this, OFDM system suffers from large PAPR. There are many approaches have been used to reduce the PAPR [7, 8]. These methods are classified into; clipping, scrambling, and coding techniques. However, these methods are easy for implementation, they may cause OOB radiation and band distortion, which would increase adjacent channel interferences and BER.

In $5 \mathrm{G}$, a filter bank multicarrier (FBMC) is proposed for OFDM limitations [9]. FBMC technique is considered as a mixture of multiplexing and modulation by splitting the wideband channel into a set of 
sub-channels narrowband channels $[10,11]$. The FBMC scheme compensates times and frequencies offset more robustly than OFDM and without CP expansion $[12,13]$. Although, FBMC provides a better spectral containment than OFDM, the interferences within and near the frequency band used will be effectively attenuated $[14,15]$.

The main contribution of this paper is improving some of challenges in wireless communication systems to follow 5G technology based of symbol time compression (STC) scheme. The STC scheme compresess OFDM symol duration into the half $[16,17]$. This paper will exploit the $50 \%$ compression of the symbol duration that is achieved by the STC scheme by extending the symbol duration without loss of the system throughput.

The proposed STC-Shaped scheme is achieved by using the interleaver-spreader and symbol shaper in the transmitter. The interleaver-spreader will rearrange the data of the compressed OFDM symbol. Thus, the whole OFDM symbol consists of four parts which are the first half of the compressed data followed by its mirror and the second half of the compressed data followed by its mirror. Then, symbol shaper will be implemented by using the proposed raised cosine function (RCF). The combination of data and its mirror with symbol shaper using will grant correctly retriving data in reciever. In the receiver side, the equalizer and despreader-combiner is achieved. The equalizer strategy is implemented via three stages; FFT, zero forcing equalization, and IFFT operation. The despreader-combiner will be combined data part with its corresponding in mirror parts to recover the compressed OFDM symbol.

The proposed scheme reduces OOB for OFDM system which is very close to that is in filter bank. In this approach, $\mathrm{CP}$ extension is eliminated without effect on the system performance compared with convential OFDM. Although, $\mathrm{CP}$ is eliminated, the system is more rubost aganist ISI in multipath environment. In this approach, PAPR is also reduced compared to conventional OFDM.

The rest of this paper is organized as follows: In Section 2, the OFDM-STC model has been introduced. The proposed model has been discussed in Section 3. Complexity analysis for proposed model has been illustrated in Section 4. In Section 5 presents the numerical results. Finally, conclusion of the proposed model has been demonstrated in Section 6 .

\section{OFDM-STC system}

The STC scheme compresses OFDM symbol into one half. The STC scheme is achieved by spreading and combining process in the transmitter and the symbol time expansion (STE) process in the receiver. The spreading and combining process in the STC transmitter is achieved as in Table 1 and the block diagram of the STC transmitter as shown in Figure $1[16,17]$.

Table 1. Spreading and combining process

\begin{tabular}{ccccccc}
\hline Transmitted bits & Polar form & Walsh code & Spreaded data & Combiner output \\
\hline 1 & $\mathrm{~b}_{1}=1$ & $\mathrm{c}_{0}=1$ & 1 & 1 & 1 & \\
0 & $\mathrm{~b}_{2}=-1$ & $\mathrm{c}_{1}=1$ & -1 & -1 & 1 & 0 \\
\hline
\end{tabular}

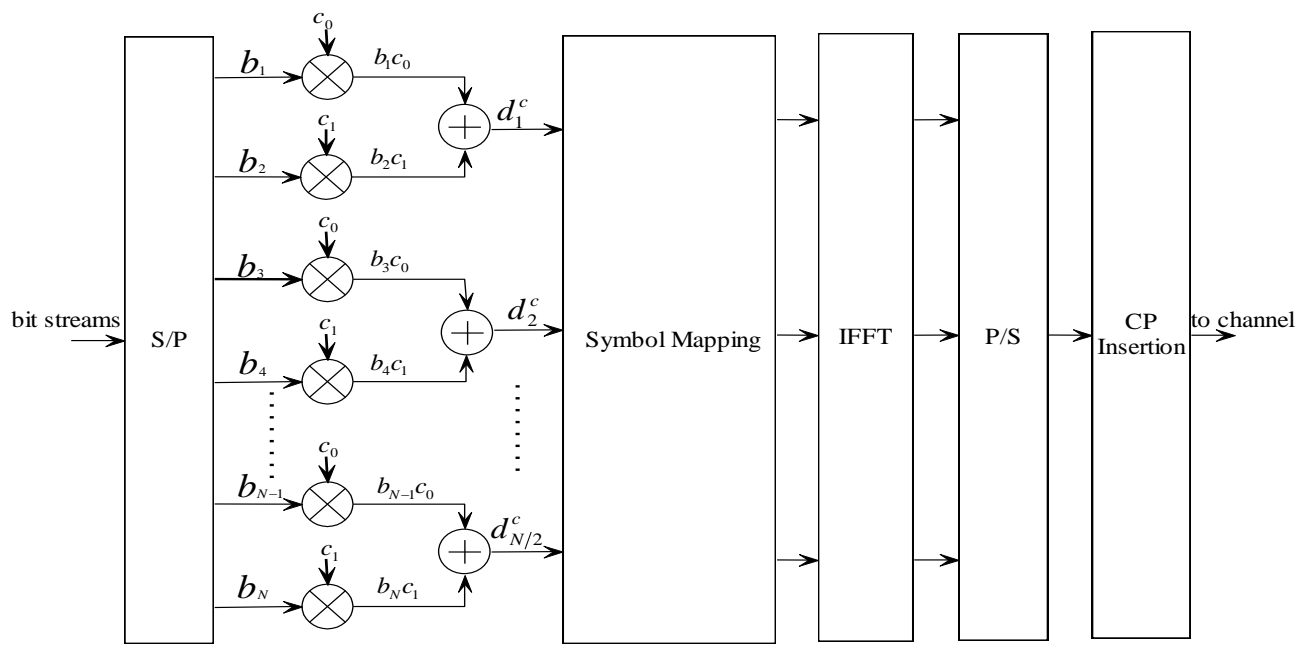

Figure 1. STC transmitter[16] 
The input data stream is recovered using STE process via despreading using the same code that is at the transmitter. The STE process will be illustrated in Table 2 and the STC receiver scheme is shown in Figure 2. Finally, the STC scheme has been reduced OFDM symbol duration to half as shown in Figure 3. This paper exploits this compression for reduction of PAPR, OOB and ISI as illustrated in the next section.

Table 2. STE process for STC

\begin{tabular}{ccccccc}
\hline Combiner output (d) & $\mathrm{dc}_{0}$ & $\hat{\mathrm{b}}_{1}=\frac{1}{2} \sum \mathrm{dc}_{0}$ & $d \mathrm{c}_{1}$ & $\hat{\mathrm{b}}_{2}=\frac{1}{2} \sum \mathrm{dc}_{1}$ \\
\hline 0 & 2 & 0 & 2 & 1 & 0 & -2 \\
\hline
\end{tabular}

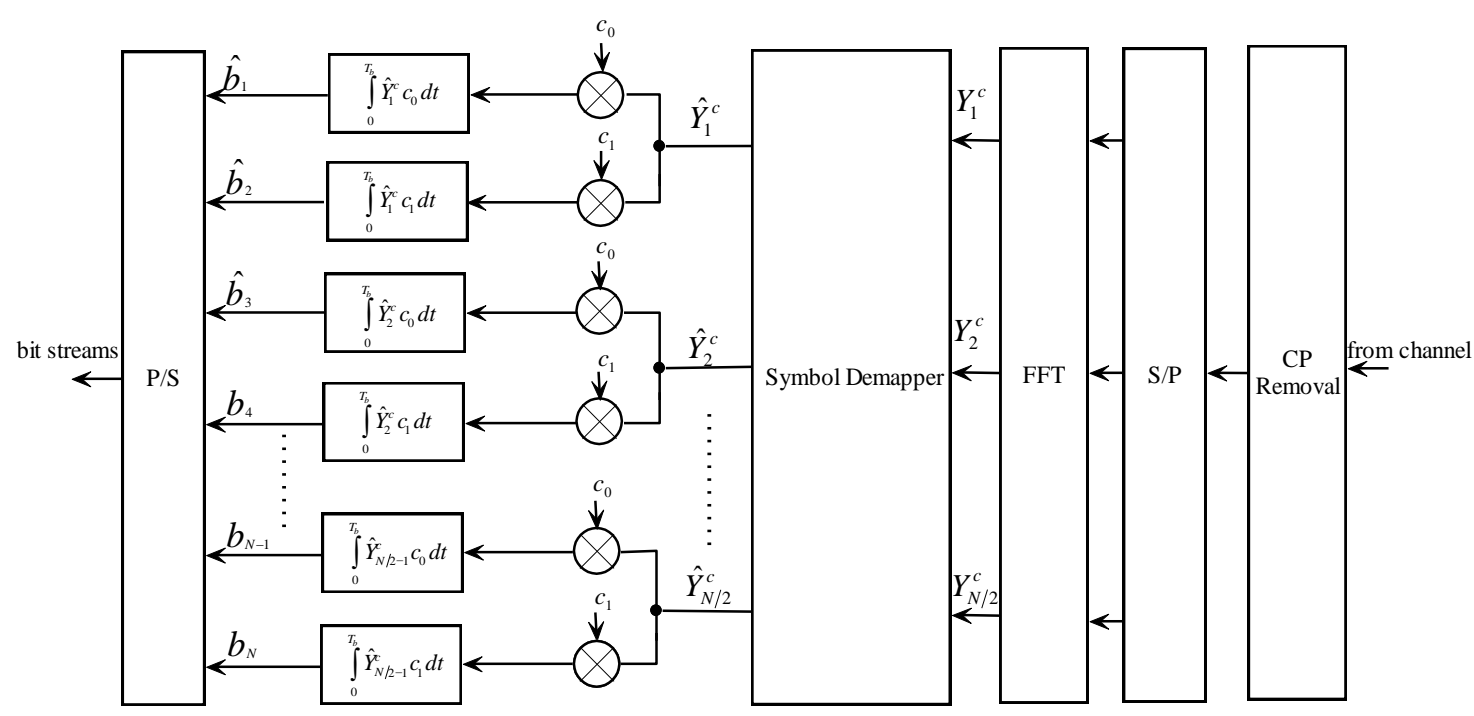

Figure 2. STC receiver [16]
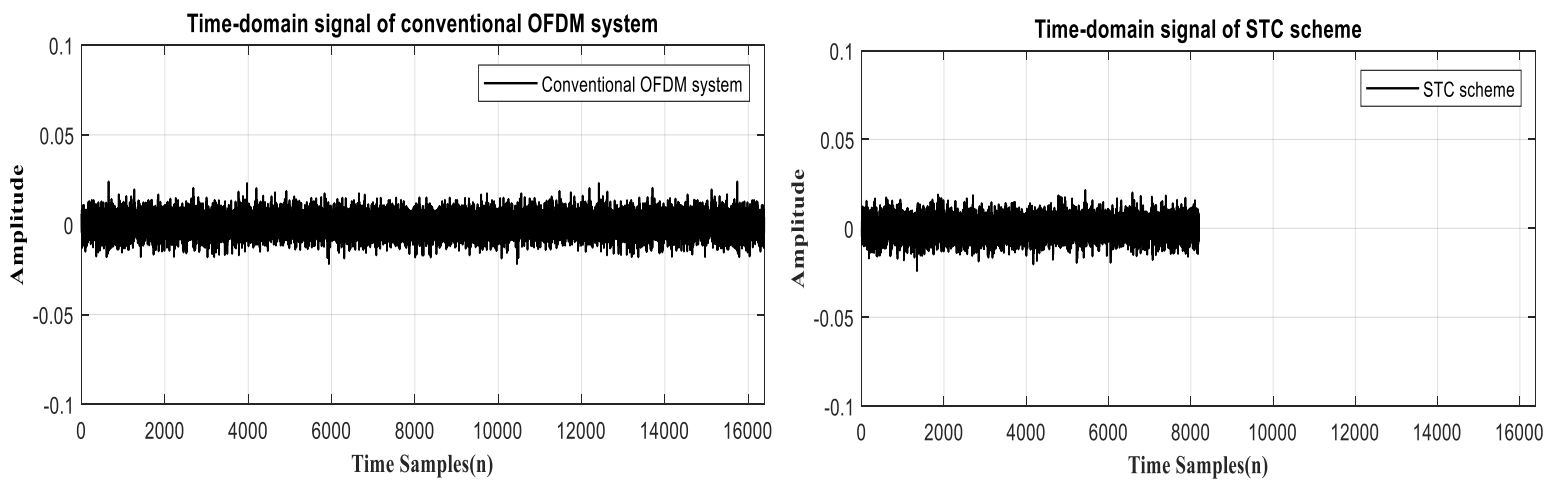

Figure 3. Time domain signal of the STC scheme and OFDM

\section{THE PROPOSED STC-SHAPED SCHEME}

The proposed STC-Shaped scheme is achieved via interleaver-spreader and symbol shaping processes at transmitter side, while combining and equalization processes at receiver side. The Block diagram of the overall proposed system is shown in Figure 4.

\subsection{STC-shaped transmitter}

The proposed system based on STC pattern reallocation in the transmitter side. The transmitter of the proposed scheme is implemented via two stages; spreader-interleaver and the symbol shaper. 


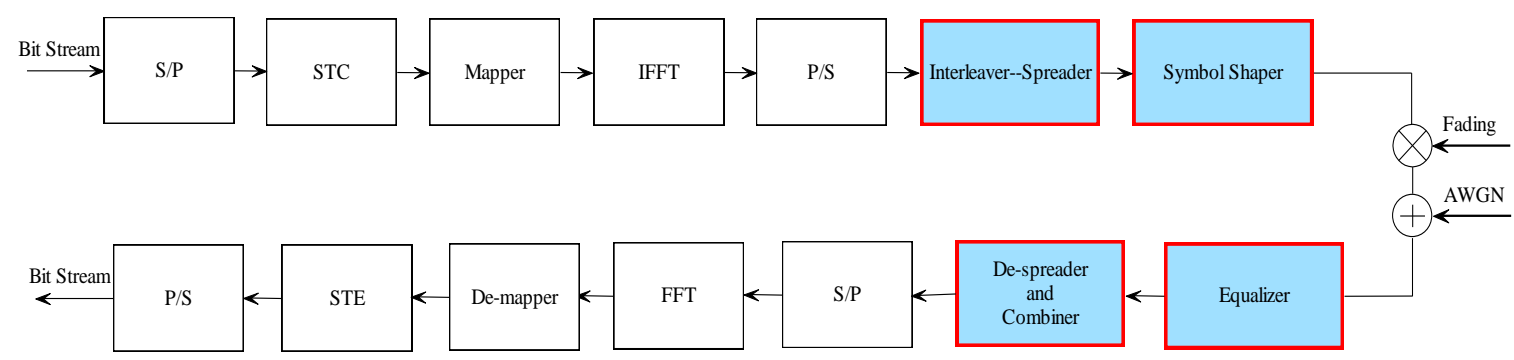

Figure 4. Block diagram of the proposed STC-Shaped System

\subsubsection{Interleaver-spreader}

This developed stage spreads the STC outputs data into $\mathrm{N}$ size similar to conventional OFDM symbol. Sorting and interleaving the sequence data is shown in Figure 5.

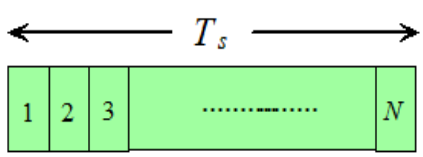

Conventional OFDM

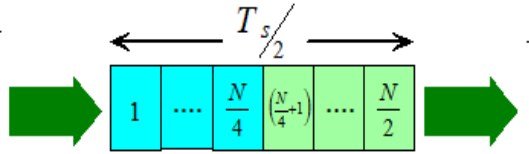

After STC process

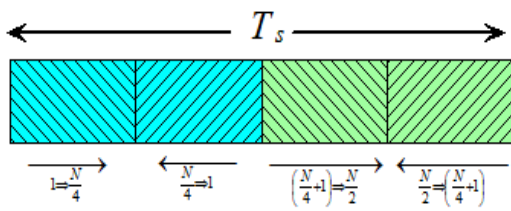

After Interleaver-Spreader

Figure 5. Interleaver-Spreader process

The mirroring arrangements grant the possibility of using raised cosine function instead of the rectangular STC. Conventional OFDM symbol with $(1 \times N)$ is compressed into half by using STC with $(1 \times N / 2)$, where $\mathrm{N}$ refers to number of subcarriers of conventional OFDM. The STC data with $A=\left[\begin{array}{lllll}A_{1} & A_{2} & \ldots & \ldots & A_{\frac{N}{2}}\end{array}\right]_{(1 \times N / 2)}$ enters to interleaver and spreader stage. The output data from interleaver-spreader is as follows:

$$
B=\left[\begin{array}{llllllllllllllllll}
A_{1} & A_{2} & \ldots & \ldots & A_{\frac{N}{4}} & A_{\frac{N}{4}} & A_{\frac{N}{4}-1} & \ldots . . & A_{1} & A_{\frac{N}{4}+1} & A_{\frac{N}{4}+2} & \ldots . . & A_{\frac{N}{2}} & A_{\frac{N}{2}} & A_{\frac{N}{2}-1} & \ldots . . & A_{\frac{N}{4}+2} & A_{\frac{N}{4}+1}
\end{array}\right]
$$

Thus, after interleaver-spreader the compressed OFDM symbol is extended to be with size $\mathrm{N}$. The vector $\mathrm{B}$ as follows: $\mathrm{B}=\left[\begin{array}{llllll}\mathrm{B}_{1} & \mathrm{~B}_{2} & \mathrm{~B}_{3} & \ldots \ldots \ldots & \mathrm{B}_{\mathrm{N}}\end{array}\right]_{(1 \times \mathrm{N})}$

\subsubsection{Symbol shaper}

The proposed raised cosine function (RCF) in (1) is suggested as follows:

$$
R C F=0.5 *\left(1-\cos \frac{2 \pi k}{N}\right), 0 \leq k \leq N-1
$$

The RCF is proposed provided that the complementary concept in the first half and the second half is achieved as shown in Figure 6.

At the first half, the first element is complement to the last element; also the second element is complement to the before last element and so on. The second half of data vector for the proposed RCF is identical to the first half. The output from symbol shaper stage is called $\mathrm{C}$ with $(1 \times N)$ which is transmitted through channel. The RCF is given by: $R=\left[\begin{array}{llllll}R_{1} & R_{2} & R_{3} & \ldots & \ldots & R_{N}\end{array}\right]_{(1 \times N)}$. The output STC-Shaped can be obtained by multiplication of each sample in $\mathrm{B}$ by corresponding coefficient in R. As, the output from symbol shaper stage as follows: $\mathrm{C}=\left[\begin{array}{lllll}\mathrm{C}_{1} & \ldots \ldots \ldots & \mathrm{C}_{\mathrm{N}}\end{array}\right]_{(1 \times \mathrm{N})}$ Where; $\mathrm{C}_{1}=\left(\begin{array}{llll}\mathrm{B}_{1} * & \mathrm{R}_{1}\end{array}\right), \cdots \mathrm{C}_{\mathrm{N}}=\left(\begin{array}{lll}\mathrm{B}_{\mathrm{N}} * & \mathrm{R}_{\mathrm{N}}\end{array}\right)$. Thus, the raised cosine multiplication with the output of the interleaver-spreader offers four benefits; OOB, PAPR, ISI reduction and increase spectrum efficiency. 


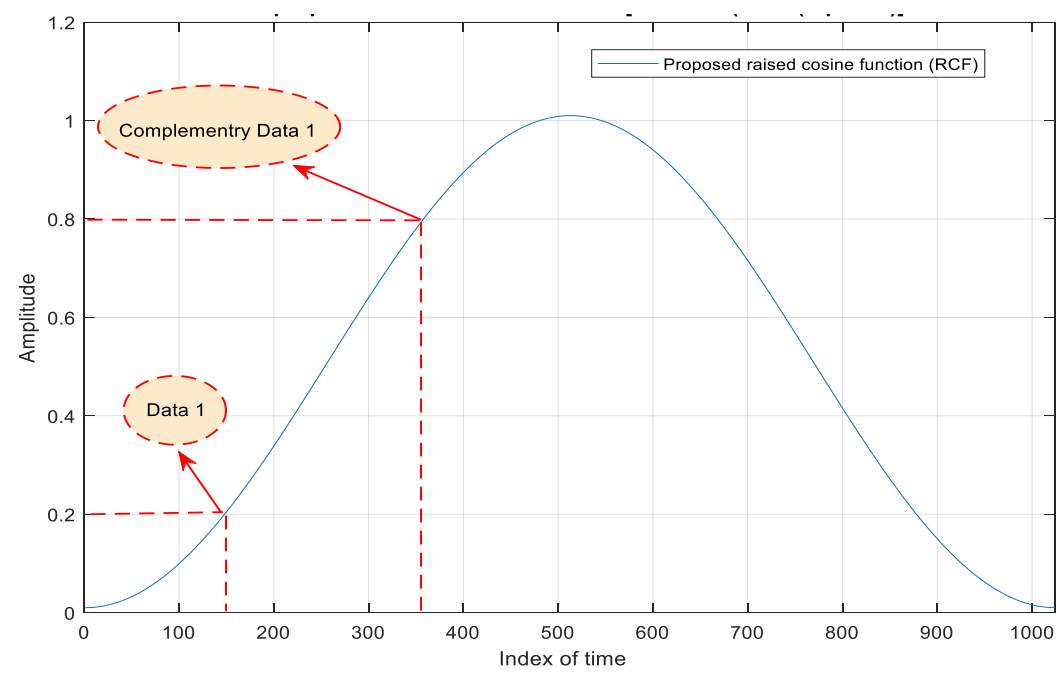

Figure 6. The proposed raised cosine function (RCF)

\subsection{STC-shaped receiver}

The proposed receiver is implemented by using two stages; channel equalizer and despreader-combiner.

\subsubsection{Channel equalization process}

The proposed system and conventional OFDM system are studied under AWGN and multipath Rayleigh fading channel environment to combat the effect of fading channel. Equalization strategy is needed for improving BER. The proposed system complexity will be slightly increased over conventional OFDM. The equalization process is shown in Figure 7.

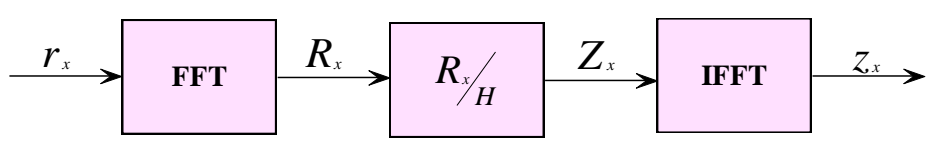

Figure 7. Equalization process

The received symbol $\left(\mathrm{r}_{\mathrm{x}}\right)$ enters into equalization stage and its output vector $\left(\mathrm{z}_{\mathrm{x}}\right)$ with $(1 \times \mathrm{N})$. The channel equalization is achieved by FFT for received symbol, zero forcing equalizer and then IFFT operation. Finally, the output data after equalization process as follows:

$$
\operatorname{IFFT}\left(Z_{\mathrm{x}}\right)=\left[\begin{array}{llllll}
Z_{1} & Z_{2} & Z_{3} & \cdots \cdots \cdots & Z_{N}
\end{array}\right]_{(1 \times N)} .
$$

\subsubsection{Despreading-combining process}

This stage is used in the receiver to recover compressed OFDM symbol. The recovery data could be obtained as follows:

$$
X=\left(Z_{1}+Z_{\frac{N}{2}}\right)\left(Z_{2}+Z_{\frac{N}{2}-1}\right) \ldots\left(Z_{\frac{N}{4}}+Z_{\frac{N}{4}+1}\right)\left(Z_{\frac{N}{2}+1}+Z_{N}\right)\left(Z_{\frac{N}{2}+2}+Z_{N-1}\right) \ldots\left(Z_{\frac{3 N}{4}}+Z_{\frac{3 N}{4}+1}\right)
$$

These yield, $X=\left[\begin{array}{llll}X_{1} & X_{2} & \cdots & X_{\frac{N}{2}}\end{array}\right]_{\left(1 \times \frac{N}{2}\right)}$ is confirming a satisfactory data recovery.

\section{COMPLEXITY ANALYSIS}

In this section, the computational complexity of the proposed STC-Shaped system is derived in terms of the number of real multiplications (RMs) and the number of real additions (RAs) compared to conventional OFDM, STC scheme, and FBMC. It is noted that, $\mathrm{N}$ is number of subcarriers and $\mathrm{K}$ is overlapped factor of FBMC. Table 3 summarizes the computational complexities for each system. 
Finally, the computational complexity for the proposed scheme is proportional $\mathrm{N} \log 2 \mathrm{~N}$, on the other hand, FBMC that is used in $5 \mathrm{G}$ proportional to $\mathrm{N}^{2}$. Thus, the proposed STC-Shaped system can follow $5 \mathrm{G}$ requirements with very low computational complexity compared to FBMC. In the next section, the performance of the proposed STC-Shaped system is achieved using matlab simulation.

Table 3. Comparison of the number of real multiplication and addition operations for various schemes

\begin{tabular}{lll}
\hline Method & number of RMs & number of RAs \\
\hline Conventional OFDM[18] & $2 \mathrm{~N} \log _{2} \mathrm{~N}-2 \mathrm{~N}$ & $3 \mathrm{~N} \log _{2} \mathrm{~N}-\mathrm{N}$ \\
STC scheme & $\mathrm{N} \log _{2} \frac{\mathrm{N}}{2}-\mathrm{N}$ & $\frac{3}{2} \mathrm{~N} \log _{2} \frac{\mathrm{N}}{2}-\frac{\mathrm{N}}{2}$ \\
FBMC[19] & $2 \mathrm{kN}^{2}+3 \mathrm{~N}$ & $2 \mathrm{~N}(\mathrm{NK}-1)+3 \mathrm{~N}+\mathrm{N}-1$ \\
Proposed system & $3 \mathrm{~N} \log _{2} \mathrm{~N}$ & $\frac{5}{2} \mathrm{~N} \log _{2} \mathrm{~N}-\frac{\mathrm{N}}{2}$ \\
\hline
\end{tabular}

\section{NUMERICAL RESULTS AND SIMULATION}

In this section, numerical results are presented to discuss the performance of the proposed STC-Shaped system. The performance of the proposed system is achieved for following parameters shown in Table 4 . In the matlab simulation a multipath Rayleigh fading channel under the COST 207 typical urban six-path (TU6) channel model is applied whose power delay profile is shown in Table 5. To illustrate the OOB power reduction, the PSD is obtaining from the power of DFT coefficients of time-domain OFDM signal [20]. The following subsections include demonstration of sidelobes suppression, PAPR and ISI reduction.

\subsection{Sidelobe suppression performances}

A comparison between conventional OFDM, FBMC and the proposed system is discussed. Figures 8(a) and (b) and Figure 9 show the PSD of the conventional OFDM, FBMC and the proposed system respectively. OFDM displays OOB radiation around $-13 \mathrm{~dB}$ as shown in Figure 8 (a) and FBMC reduces PSD about $-100 \mathrm{~dB}$ as shown in Figure 8(b). The reduction of OOB for FBMC is costing of increasing complexity. The proposed STC-Shaped filter significantly reduces OOB compared to conventional OFDM. The proposed system decreases $\mathrm{OOB}$ by about $-70 \mathrm{~dB}$. Thus, the $\mathrm{OOB}$ of proposed scheme is very close to that is in FBMC. The proposed scheme has lower complexity than FBMC. Hence, the proposed scheme can follow the FBMC with lower complexity. Also, the proposed STC- Shaped scheme is without CP insertion and hence its BW efficiency is improved.

Table 4. imulation parameters

\begin{tabular}{ll}
\hline Parameters & Specification \\
\hline Conventional OFDM subcarriers $N$ & 1024 \\
CP length $N_{\text {CP_in_conventional_OFDM }}$ & 256 \\
CP length in proposed system & None \\
Modulation type & BPSK \\
Overlapped factor K in FBMC & 4 \\
No of OFDM symbols & 10000 \\
\hline
\end{tabular}

Table 5. Power delay profile [21]

\begin{tabular}{cccccccc}
\hline & Tap 1 & Tap 2 & Tap 3 & Tap 4 & Tap 5 & Tap 6 & Unit \\
\hline Delay & 0 & 0.2 & 0.5 & 1.6 & 2.3 & 5 & $(\mu \mathrm{sec})$ \\
Power & -3 & 0 & -2 & -6 & -8 & -10 & $(\mathrm{~dB})$ \\
\hline
\end{tabular}
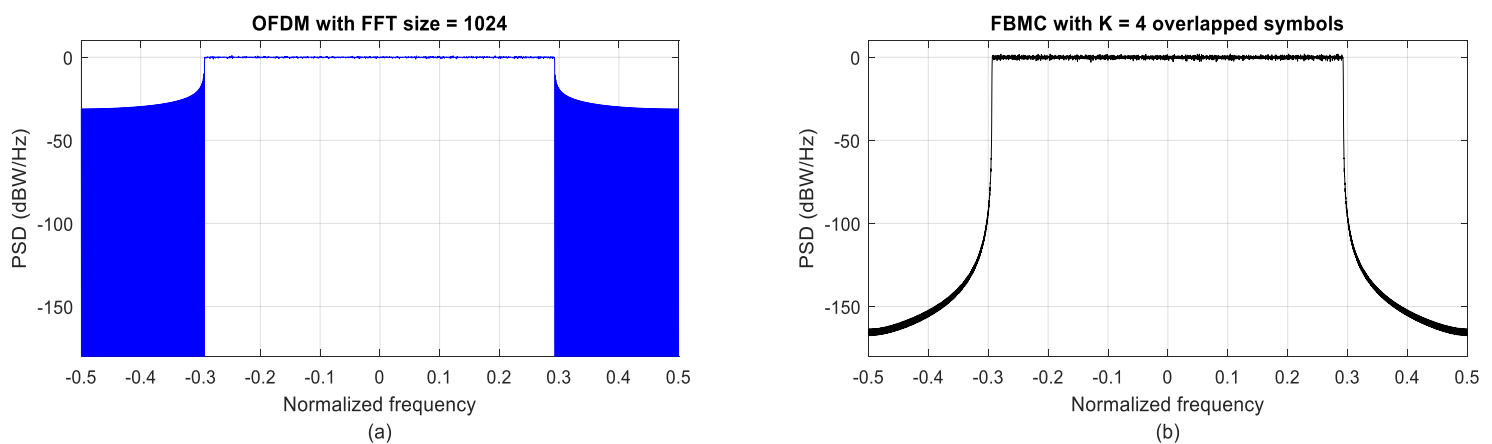

Figure 8. (a) PSD of OFDM and (b) PSD of FBMC [22, 23] 


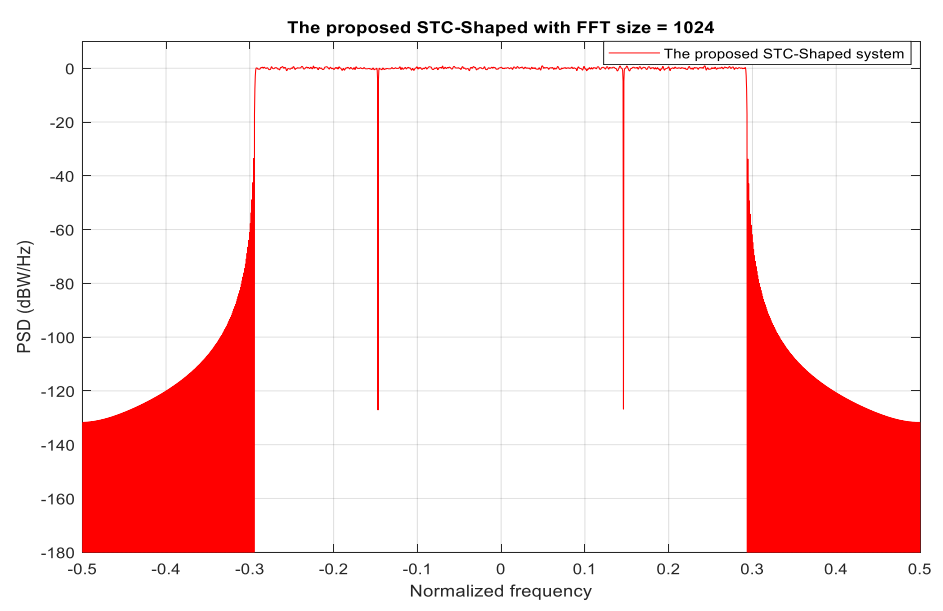

Figure 9. PSD of the proposed STC-Shaped System

\subsection{PAPR performance}

The OFDM multicarrier systems limitation is the higher peaks of the added coherently non-constant envelops [24]. The OFDM signal has $\mathrm{N}$ subcarriers are added together. If $\mathrm{N}$ is sufficiently large, the resulting signal will be close to a complex Gaussian model [25]. This implies that Gaussian is distributed in both its real and imaginary parts, and its envelope and energy follows respectively Rayleigh and exponential distributions. PAPR is articulated as a discrete version of $\mathrm{X}[\mathrm{n}]$ using $(2)[26,27]$.

$$
\operatorname{PAPR}(x[n])=\max _{0 \leq n \leq N-1} \frac{|x[n]|^{2}}{E\left[|x[n]|^{2}\right]}
$$

Figure 10(a) shows eight sinusoidal harmonic signals without shaping, whereas a Figure 10(b) declares how the high peaks are reduced by using the raised cosine function for eight sinusoidal signals with different frequencies. Figure 10(c) illustrates how a high peak is achieved by consistently adding eight sinusoidal signals with distinct frequencies and phase shifts. The resulting signal envelope shows elevated peaks when simultaneously elevated peaks are aligned with the instantaneous amplitudes of the distinct signals. Such elevated peaks will result in signal excursions into the transmitter power amplifier nonlinear area of operation, resulting in nonlinear distortions and spectral spread.
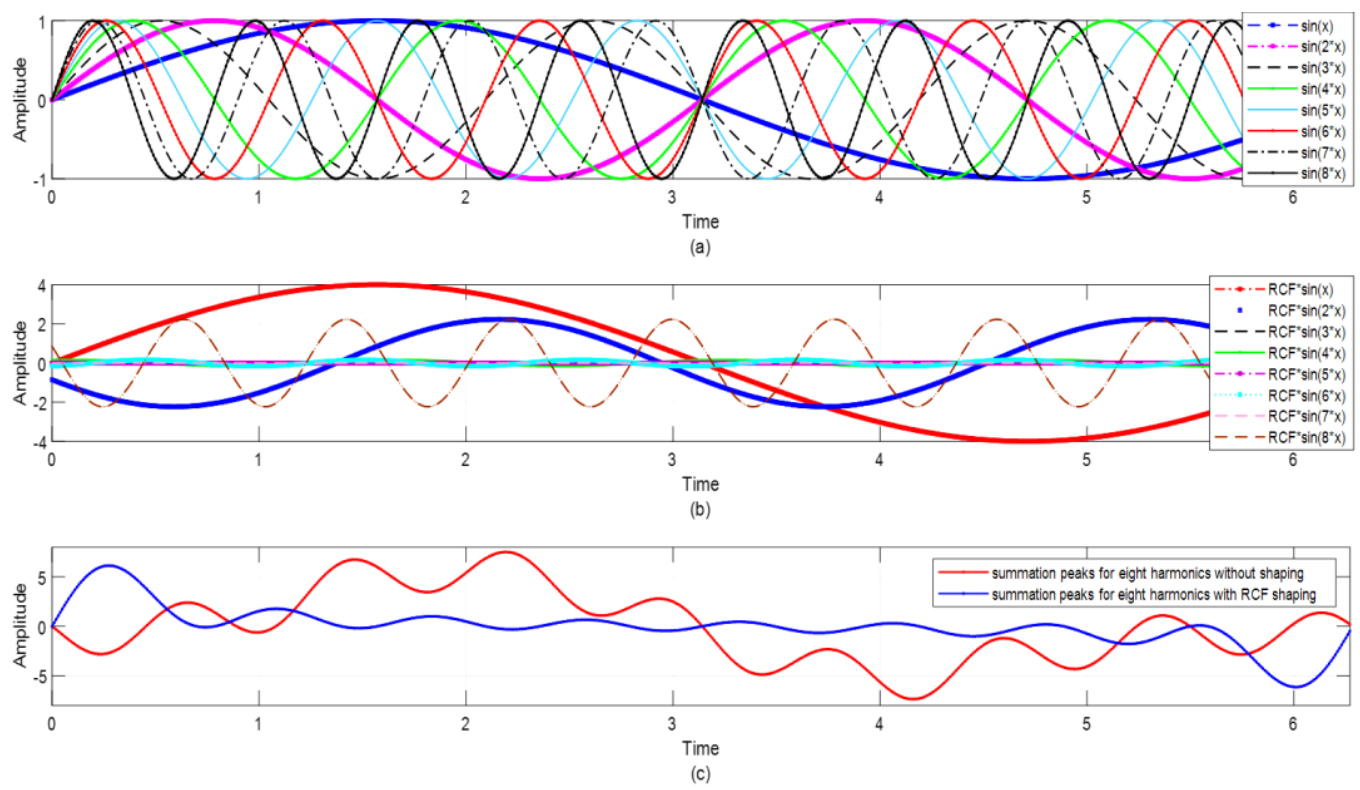

Figure 10. (a) Harmonic signal without shaping, (b) with shaping and

(c) High peaks generated by summing multiple sinusoids 
From Figure 10, PAPR is enhanced by using the proposed shaping, where PAPR with shaping equal $6.3365 \mathrm{~dB}$ but without shaping equal $9.7318 \mathrm{~dB}$ from calculation when 2 is applied. Thus, the proposed STC scheme improves the PAPR by about $3 \mathrm{~dB}$. This is also derived by computing cumulative distribution function $(\mathrm{CDF})$ of conventional OFDM. Figure 11 shows CDF of the proposed STC- Shaped system and conventional OFDM systems. CDF plot is shown in Figure 11 with the probability $\operatorname{Pr}(\mathrm{Z}<=\mathrm{z})$ versus PAPR $(\mathrm{dB})$. For a probability of 0.4 , PAPR values for proposed system and OFDM are $8 \mathrm{~dB}$ and $11 \mathrm{~dB}$. It has PAPR performance that refers to as $3 \mathrm{~dB}$ better than the OFDM.

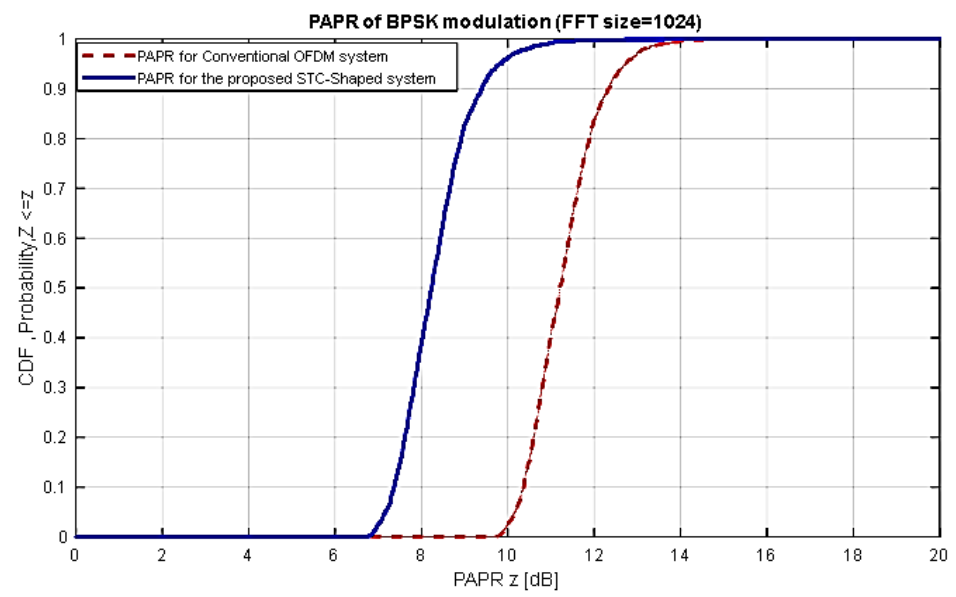

Figure 11. PAPR reduction for the proposed STC- Shaped system and conventional OFDM system

\subsection{BER performance}

The comparison and evaluation between FBMC and OFDM systems in term of BER using the proposed scheme and conventional OFDM schemes which is under AWGN channel is shown in Figure 12. The result of the proposed system and conventional OFDM-CP elimination under AWGN channel gives the same performance as shown in Figure 12. Figure 13 Shows the BER performance of the proposed STC-Shaped system compared with conventional OFDM theoretical and conventional OFDM-CP elimination under Rayleigh channel. The proposed system has better BER performance than conventional OFDM-CP elimination in multipath fading as shown in Figure 13. Thus, the proposed scheme is more robust against ISI than conventional OFDM-CP elimination.

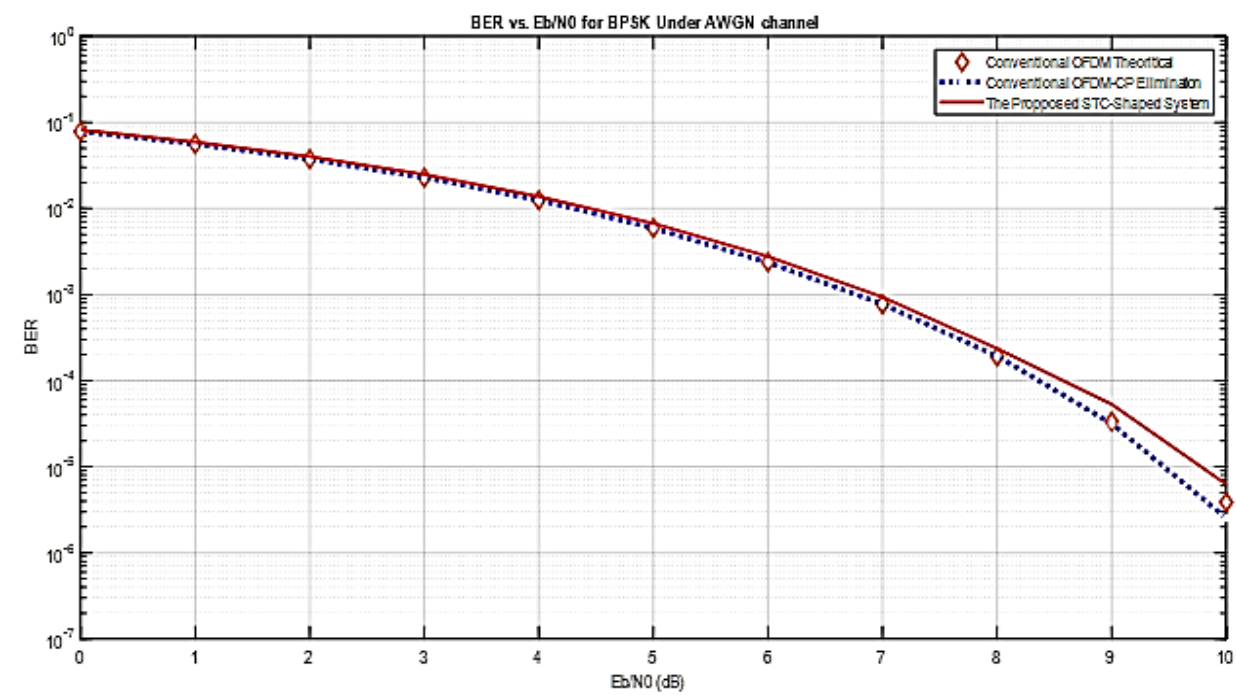

Figure12. BER performance of the proposed STC-Shaped system under AWGN channel 


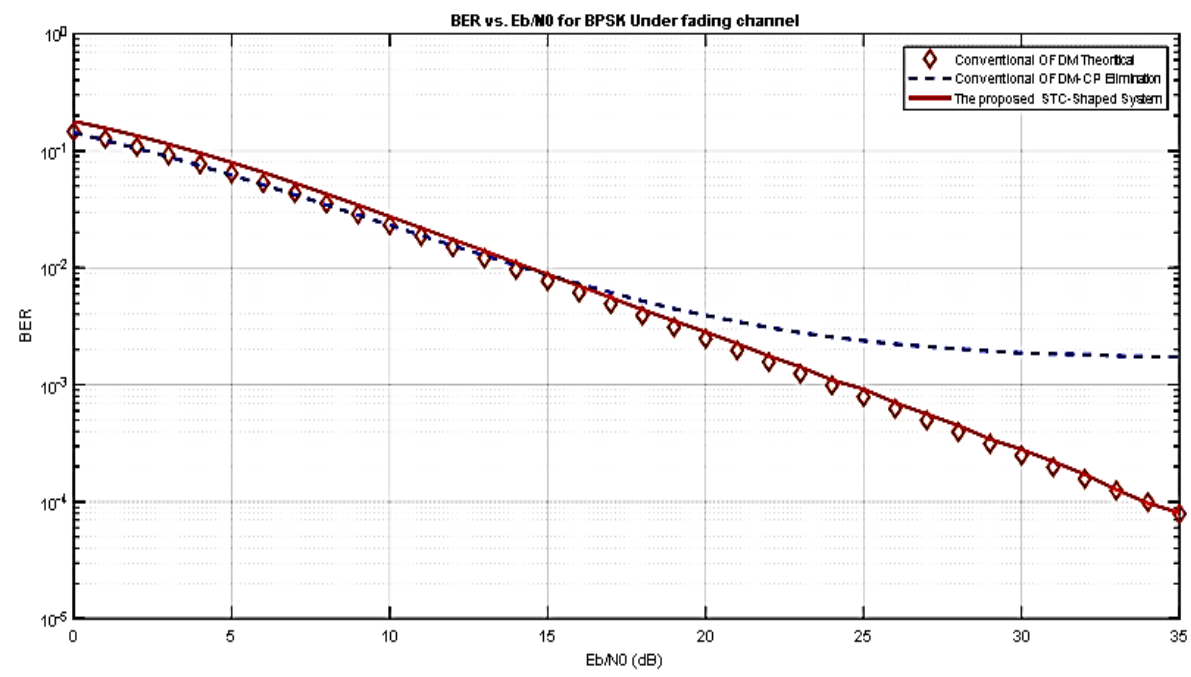

Figure 13. BER performance of the proposed STC-Shaped system under Rayleigh channel

\section{CONCLUSION}

An efficient technique for $5 \mathrm{G}$ mobile communications performance has been proposed. The proposed system is based on STC scheme. The proposed STC-Shaped scheme is achieved using spreader-interleaver and symbol shaper processes in the transmitter side. Meanwhile, equalization and despreader-combiner processes are applied in the receiver side. Also, the proposed scheme is evaluated using simulation compared to conventional OFDM-CP elimination and FBMC. The numerical results show that the OOB of the proposed scheme is reduced by about-70dB which is very close to FBMC and PAPR of the proposed system is reduced by $3 \mathrm{~dB}$ compared to conventional OFDM. In addition to this, the proposed system is more robust against to ISI although CP is eliminated. Due to CP elimination, the proposed scheme increases BW efficiency. Finally, the computational complexity of the proposed system is first order polynomial while FBMC is second order polynomial. Thus, the proposed scheme has approximately the same performance that is required in $5 \mathrm{G}$ systems but with very low computational complexity.

\section{REFERENCES}

[1] Gupta, Akhil, and Rakesh Kumar Jha, "A survey of 5G network: Architecture and emerging technologies," IEEE access, vol. 3, 1206-1232, 2015.

[2] Tao, Yunzheng, et al., "A survey: Several technologies of non-orthogonal transmission for 5G," China communications, vol. 12, no. 10, pp. 1-15, 2015.

[3] Pietikäinen, Kari, "Orthogonal frequency division multiplexing," Internet presentation, 2005.

[4] Li, Xiaodong, Constantinos Basil Papadias, and Harish Viswanathan, "Orthogonal frequency division multiplexing transmit diversity system for frequency-selective fading channels," U.S. Patent No. 7,020,072, 2006.

[5] K. Hussain, A. Lojo, and R. López-Valcarce, "Flexible spectral precoding for sidelobe suppression in OFDM systems," IEEE International Conference on Acoustics, Speech and Signal Processing, pp. 4789-4793, 2019.

[6] Mizutani, Yuji, et al., "A Low-pass Filtered Time-domain Window for DFTs-OFDM to Reduce Out-of-band Emission with Low Complexity," 16th IEEE Annual Consumer Communications and Networking Conference, pp. 1-2, 2019.

[7] Lin, Shu-Ping, Yung-Fang Chen, and Shu-Ming Tseng, "Iterative smoothing filtering schemes by using clipping noise-assisted signals for PAPR reduction in OFDM-based carrier aggregation systems," IET Communications, vol. 13, no. 6, pp. 802-808, 2019.

[8] Lu, Shixian, Daiming Qu, and Yejun He, "Sliding window tone reservation technique for the peak-to-average power ratio reduction of FBMC-OQAM signals," IEEE Wireless Communications Letters, vol. 1, no. 4, pp. 268-271, 2012.

[9] Mahama, Sumaila, et al., "Iterative interference cancellation in FBMC-QAM systems," IEEE Wireless Communications and Networking Conference, 2019.

[10] Zhao, Zhao, Nikola Vucic, and Malte Schellmann, "Transmitter, receiver and system for filterbank multicarrier communication," U.S. Patent No. 10,355,904, 2019.

[11] U. C. Samal, B. Appasani, and D. Kumar Mohanta, "5G Communication Networks and Modulation Schemes for Next-Generation Smart Grids," Smart Grids and Their Communication Systems, pp. 361-399, 2019.

[12] Wunder, Gerhard, et al., "5GNOW: Non-orthogonal, asynchronous waveforms for future mobile applications," IEEE Communications Magazine, vol. 52, no. 2, pp. 97-105, 2012. 
[13] Ibrahim, A. N., and M. F. L. Abdullah, "The potential of FBMC over OFDM for the future 5G mobile communication technology," AIP Conference Proceedings, vol. 1883, no. 1, 2017.

[14] Farhang-Boroujeny, Behrouz, "Filter bank multicarrier modulation: A waveform candidate for 5G and beyond," Advances in Electrical Engineering, vol. 2014, 2014.

[15] Rachini, Ali S., and Mustafa Musa Jaber. "Performance of FBMC in 5G Mobile Communications Over Different Modulation Techniques," International Symposium on Networks, Computers and Communications, 2019.

[16] El-Bakry, Mohammed S., Hamed A. El-Shenawy, and Abd El Hady A. Ammar, "A symbol time compression for ICI reduction in high mobility OFDM systems," 29th International Conference on Microelectronics, 2017.

[17] El-Bakry, Mohammed S., Hamed A. El-Shenawy, and Abd El-Hady A. Ammar, "A time inversion and symbol time compression (TI-STC) scheme for ICI cancellation in high mobility OFDM systems," Japan-Africa Conference on Electronics, Communications and Computers, 2017.

[18] Fuqin Xiong, "Digital Modulation Techniques," Second Edition, Artech House, 2006.

[19] Husam, Al-amaireh, and Zsolt Kollár, "Complexity Comparison of Filter Bank Multicarrier Transmitter Schemes," 11th International Symposium on Communication Systems, Networks \& Digital Signal Processing, 2018.

[20] Pan, Lebing, Jun Ye, and Xiaobing Yuan, "Spectral Precoding for Out-of-Band Power Reduction Under Condition Number Constraint in OFDM-Based Systems," Wireless Personal Communications, vol. 95, no. 2, pp. 1677-1691.

[21] Kun-Yi Lin, Hsin-Piao Lin and Ming-Chien Tseng," An Equivalent Channel Time Variation Mitigation Scheme for ICI Reduction in High-Mobility OFDM Systems," IEEE transactions on broadcasting, vol. 58, no. 3, pp.472-479, 2012.

[22] Nagul, Shribala, "A review on 5G modulation schemes and their comparisons for future wireless communications," Conference on Signal Processing And Communication Engineering Systems, 2018.

[23] Kundrapu, Srikanth, et al., "Characteristic Analysis of OFDM, FBMC and UFMC Modulation Schemes for Next Generation Wireless Communication Network Systems," 3rd International conference on Electronics, Communication and Aerospace Technology, 2019.

[24] Kundu, Poonam, and Prabhjot Kaur, "Comparison of peak to average power reduction techniques in OFDM," International Conference on Advances in Computing, Communications and Informatics, 2014.

[25] V. Tarokh and H. Jafarkhani, "On the computation and reduction of the peak-to-average power ratio in multicarrier communications," IEEE Trans. Commun., vol. 48, pp. 37-44, 2000.

[26] Rahmatallah, Yasir, and Seshadri Mohan, "Peak-to-average power ratio reduction in OFDM systems: A survey and taxonomy," IEEE communications surveys \& tutorials, vol. 15, no. 4, pp. 1567-1592, 2013.

[27] Mahadevaswamy, U. B., and M. N. Geetha, "A comparative survey on PAPR reduction in OFDM signal," International Conference on Electrical, Electronics, Communication, Computer and Optimization Techniques, 2016.

\section{BIBLIOGRAPHY OF AUTHORS}

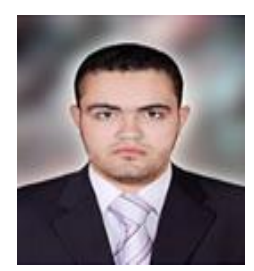

Mohamed Yasin is an Assistant Lecturer in the Faculty of Engineering, Al-Azhar University, Cairo, Egypt. He received his BSc and MSc in Electronics and Communications Engineering from the Faculty of Engineering, Al-Azhar University, Cairo, Egypt, in 2011 and 2017 respectively. He is currently a PhD student at Faculty of Engineering, Al-Azhar university, Cairo. His research activities are within wireless communications and communication networks.

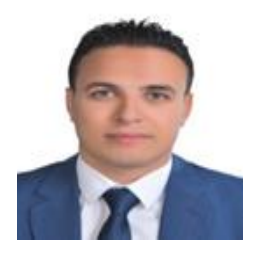

Mohamed S. Elbakry received his BSc. in electronics and communications engineering from Institute of Aviation Engineering and Technology (IAET), Giza, Egypt, in 2005 and his MSc. degree and $\mathrm{PhD}$ from faculty of engineering, Al-Azhar University, Cairo, Egypt, in 2014 and 2018 respectively. He is an Assistant professor at IAET. His research interests include OFDM systems, interference cancellation, channel estimation and signal processing for $5 \mathrm{G}$.

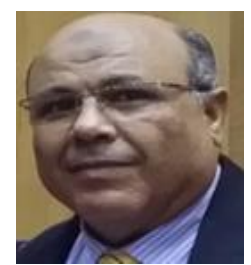

El-Sayed Soliman is an Associate Prof. of Power Electronics, Faculty of Engineering, Al-Azhar University, Egypt. Head of the Research and Development Center at Al-Ahsa College of Technology, Saudi Arabia since 2003 up to 2013. His research interest is in area of microcomputer-based systems, secure universal RTC embedded systems, IoT, interfacing green energy resources with public grid. He is much interested by planning, promoting and implementing the giftedness programs and strategies.

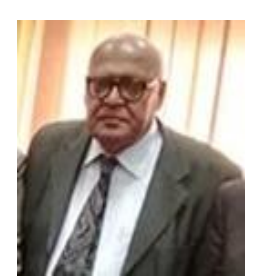

Abdelhady Abdelazim Ammar is a professor in Electronics and Communications Engineering department, Faculty of Engineering, Al-Azhar University, Cairo, Egypt since 1988. His research activities are within digital communications, mobile communications and digital signal processing. 